\title{
Detection of interventricular dyssynchrony: An evolution of the phase analysis technique
}

\author{
Saurabh Malhotra, MD, MPH, FACC, FASNC, ${ }^{a}$ and Diwakar Jain, MD, FASNC ${ }^{b}$ \\ a Division of Cardiovascular Medicine, Jacob School of Medicine and Biomedical Sciences, \\ University at Buffalo, Buffalo, NY \\ b Section of Cardiovascular Medicine, New York Medical College, Westchester Heart and \\ Vascular, Westchester Medical Center, Valhalla, NY
}

Received May 16, 2016; accepted May 17, 2016

doi: $10.1007 / \mathrm{s} 12350-016-0550-z$

\section{See related article, pp. 1680-1686}

Ventricular dyssynchrony is prevalent among patient with heart failure (HF) and portends poor prognosis. ${ }^{1,2}$ Ventricular dyssynchrony can manifest itself in numerous forms - atrioventricular (AV) dyssynchrony, interventricular (between the ventricles) dyssynchrony, and intraventricular (within the ventricle) dyssynchrony. While AV dyssynchrony is easily correctable with pacemakers, the complex pathophysiology of both interventricular and intraventricular dyssynchrony makes management more challenging. Interventricular dyssynchrony can be easily recognized as either a right (RBBB) or a left bundle branch block (LBBB) on surface electrocardiogram (ECG). Typically, electrical activation in LBBB has a U-shaped pattern that begins at the septum, courses through the apex, with delayed activation of the lateral and posterolateral portions of the left ventricle $(\mathrm{LV}){ }^{3}$ In patients with $\mathrm{RBBB}$, the earliest ventricular activation occurs on the left ventricular side of the interventricular septum, after a considerable delay arrives to the right ventricular (RV) side of the septum with activation of the remainder of the ventricle even later-RV anterior wall activation followed by lateral wall and the outflow tract. ${ }^{4}$ This activation pattern in RBBB (early activation of septum and delayed

Reprint requests: Saurabh Malhotra, MD, MPH, FACC, FASNC, Division of Cardiovascular Medicine, Jacob School of Medicine and Biomedical Sciences, University at Buffalo, 875 Ellicott Street, Clinical and Translation Research Center, Suite 7030, Buffalo, NY, 14221; drsmalhotra@hotmail.com

J Nucl Cardiol 2017;24:1687-9.

1071-3581/\$34.00

Copyright (C) 2016 American Society of Nuclear Cardiology. activation of lateral wall) essentially mirrors the activation pattern in LBBB. Despite dyssynchronous electrical activation in both, improvement with resynchronization is only noted among those with LBBB. ${ }^{5}$

The prevalence of intraventricular dyssynchrony (primarily LV) is now being increasing recognized. Although it cannot be measured on surface ECG, imaging modalities like echocardiography (e.g., strain imaging) and radionuclide imaging approaches (e.g., phase analysis of gated SPECT/PET and ERNA) provide an opportunity to assess such a phenomenon. Gated SPECT and PET are now established techniques to determine the presence of intra-left-ventricular dyssynchrony, and gender-specific cut-offs for the presence of left intraventricular dyssynchrony are available from gated SPECT studies. ${ }^{6}$ Previously, an equilibrium radionuclide angiography (ERNA) study had shown that intraventricular dyssynchrony is more closely related to cardiac performance, than interventricular dyssynchrony. ${ }^{7}$ In a study of 103 patients with dilated cardiomyopathy, $25 \%$ of whom had LBBB, Fauchier et al studied the relationship between inter- and intraventricular dyssynchrony on ERNA, with cardiac hemodynamic status and cardiac events (cardiac death and worsening HF). Increasing left and right intraventricular dyssynchrony correlated with a lower LV ejection fraction and cardiac index, while interventricular dyssynchrony was not related to these hemodynamic parameters. Additional studies have also shown a strong relationship between left intraventricular dyssynchrony with HF outcomes and arrhythmic events, ${ }^{2,8}$ though there is paucity of studies on right intraventricular dyssynchrony.

Despite these observations, novel imaging approaches that measure intraventricular dyssynchrony, left ventricular in particular, have failed to show any benefit from dyssynchrony-guided resynchronization. ${ }^{9}$ Thus 
currently, electrical interventricular dyssynchrony with a LBBB pattern is widely accepted as the strongest predictor of improvement in cardiac function after resynchronization. ${ }^{10}$ However, RBBB is also recognized as not being completely benign. ${ }^{11}$ Furthermore, studies have also shown that those with RBBB and concomitant left ventricular conduction abnormalities may also benefit with CRT. ${ }^{12,13}$ Although surface ECG is routinely used to determine cardiac conduction abnormalities, it may not completely represent the activation of the myocardial substrate in its entirety. ${ }^{14}$ ECG may not be able to capture the severity of the electrical delay in all segments and regional variations in small electrical vectors may not be captured. Interstitial fibrosis and consequent heterogeneous depolarization produce variations in regional loading conditions that result in mechanical dyssynchrony that may be better represented by mechanical activation than by electrical activation. Hence, a different approach to measure interventricular dyssynchrony could involve the assessment of the timing of myocardial contractility. Although this has been previously performed using ERNA, ${ }^{7,15}$ the wider use of gated SPECT makes it a more attractive approach.

In the current issue of the journal, Zhou et al report the application of phase analysis of gated SPECT to develop a tool for measuring interventricular dyssynchrony. ${ }^{16}$ They further report whether interventricular dyssynchrony measured by their tool relates to ECG evidence of interventricular conduction delay. The authors performed a retrospective study of 61 patients with dilated cardiomyopathy who underwent both a 12lead ECG and a gated Tc-99m SPECT. All the patients included in this analysis had either LBBB or RBBB on ECG, and all had Tc-99m uptake in the RV on SPECT. Various LV and RV parameters (base, apex, myocardial radius, and the center of the ventricle) were manually identified from their respective images in short-axis orientation. An automated sampling algorithm searched for the maximal circumferential count profiles in a 3dimensional fashion, separately for the LV and RV. First-harmonic Fourier transformation, of the circumferential count profiles was performed by the phase analysis technique to yield the timing of the onset of contraction (phase, in degrees) of each myocardial sample. This is a standard approach of assessing the timing of ventricular contractility by phase analysis. The $\mathrm{LV}$ and RV phase profiles were segmented using a 13segment model and mean phase for each of the LV and RV segments were determined. The difference between the mean phase of the mid LV free wall and the mid LV septum constituted the LV contraction delay, while the difference between the mid RV free wall and mid RV septum represented the RV contraction delay. Interventricular conduction delay was defined as follows: (a) difference in $\mathrm{LV}$ contraction $>$ difference in $\mathrm{RV}$ contraction $\left(\Delta_{\mathrm{LV}}>\Delta_{\mathrm{RV}}\right)$, signified the LV to contract after the RV.

(b) difference in $\mathrm{LV}$ contraction $<$ difference in $\mathrm{RV}$ contraction $\left(\Delta_{\mathrm{LV}}<\Delta_{\mathrm{RV}}\right)$, signified the LV to contract earlier than the RV.

Concordance between interventricular contraction delay by gated SPECT and interventricular conduction delay on ECG was determined for all patients. In a predominantly male population $(85 \%)$, with a mean LV ejection fraction of $28 \%$ and mean QRS duration of 163 milliseconds (ms), LBBB was present in 30 and RBBB in 31 patients. Among patients with LBBB, 90\% (27 of 30) had $\Delta_{\mathrm{LV}}>\Delta_{\mathrm{RV}}$, and among the RBBB patients $84 \%$ (26 of 31) had $\Delta_{\mathrm{LV}}<\Delta_{\mathrm{RV}}$, with a good overall agreement of $87 \%$ and a Kappa statistic of $74 \%$.

This study extends the applicability of the phase analysis technique of timing left intraventricular contraction, to timing right intraventricular contraction, and provides the ability to measure the temporal differences in the contraction of the two ventricles. The results presented in this analysis simply show that electrical conduction differences across the ventricles can be identified as either being LBBB or RBBB, based on the timing of contractility/thickening of the LV or RV segments on phase analysis, and thus proves what is evident on a regular surface ECG. The study lacks validation of the measured differences in a normal population without any bundle branch block, which would allow the user of this technique to separate a true bundle branch block from noise. It is important to note that all patients in this study had either LBBB or RBBB. Whether this tool will perform in a similar fashion among those with HF, but without either form of bundle branch block and identify dyssynchrony that does not confine to these two electrical patterns needs to be evaluated. This is critical, as observations have shown (echocardiographic and radionuclide imaging) that not all patients with LBBB may be dyssynchronous, despite a septal to lateral contraction delay. ${ }^{17,18}$ Furthermore, it is likely that many patients with HF and no bundle branch block could have mechanical, and perhaps electrical, activation in a manner similar to those with a bundle branch block. ${ }^{17}$ This will have profound implications, as a subpopulation without electrical LBBB could have a similar degree of improvement with resynchronization if mechanical contraction parallels a LBBB pattern. In addition to the identification of contraction pattern, it is also important to account for confounding caused by the presence of scar, as an area of scar will be the last to contract regardless of the electrical bundle pattern, and this was not accounted for in this study. Furthermore, this tool mandates tracer 
uptake in the RV-which was present in among all patients included in this highly selected population. Despite cardiomyopathy, many patients may not have $\mathrm{RV}$ uptake and this may limit the effectiveness of this tool.

Nonetheless, this novel tool does open the applicability of assessing interventricular dyssynchrony by gated SPECT - the most widely used cardiac radionuclide imaging technique. In a LBBB population, this tool will need prospective evaluation comparing CRT response stratified by the presence of electrical versus mechanical LBBB. It has to be kept in mind that conventional CRT is designed and implanted in a fashion to reduce septal to lateral wall delay in the $L V$, and thus can only correct dyssynchrony caused by LBBB and not by other conduction abnormalities. Thus, it is plausible that the lack of improvement with CRT in other conduction abnormalities is actually a limitation of the CRT itself and does not disprove the utility of dyssynchronyguided resynchronization. The real value of this tool developed by Zhou et al will only come to fruition when the electrophysiology field matures to develop the ability to correct non-LBBB dyssynchrony, perhaps with multisite pacing.

\section{References}

1. AlJaroudi W, Alraies MC, Menon V, Brunken RC, Cerqueira MD, Jaber WA. Predictors and incremental prognostic value of left ventricular mechanical dyssynchrony response during stress-gated positron emission tomography in patients with ischemic cardiomyopathy. J Nucl Cardiol 2012;19:958-69.

2. Aljaroudi WA, Hage FG, Hermann D, et al. Relation of left-ventricular dyssynchrony by phase analysis of gated SPECT images and cardiovascular events in patients with implantable cardiac defibrillators. J Nucl Cardiol 2010;17:398-404.

3. Poole JE, Singh JP, Birgersdotter-Green U. QRS duration or QRS morphology: What really matters in cardiac resynchronization therapy? J Am Coll Cardiol 2016;67:1104-17.

4. Auricchio A, Lumens J, Prinzen FW. Does cardiac resynchronization therapy benefit patients with right bundle branch block: Cardiac resynchronization therapy has a role in patients with right bundle branch block. Circ Arrhythm Electrophysiol 2014;7:532-42.

5. Zareba W, Klein H, Cygankiewicz I, et al. Effectiveness of cardiac resynchronization therapy by QRS morphology in the multicenter automatic defibrillator implantation trial-cardiac resynchronization therapy (MADIT-CRT). Circulation 2011;123:1061-72.
6. Chen J, Garcia EV, Folks RD, et al. Onset of left ventricular mechanical contraction as determined by phase analysis of ECGgated myocardial perfusion SPECT imaging: Development of a diagnostic tool for assessment of cardiac mechanical dyssynchrony. J Nucl Cardiol 2005;12:687-95.

7. Fauchier L, Marie O, Casset-Senon D, Babuty D, Cosnay P, Fauchier JP. Interventricular and intraventricular dyssynchrony in idiopathic dilated cardiomyopathy: A prognostic study with fourier phase analysis of radionuclide angioscintigraphy. J Am Coll Cardiol 2002;40:2022-30.

8. Friehling M, Chen J, Saba S, et al. A prospective pilot study to evaluate the relationship between acute change in left ventricular synchrony after cardiac resynchronization therapy and patient outcome using a single-injection gated SPECT protocol. Circ Cardiovasc Imaging 2011;4:532-9.

9. Ruschitzka F, Abraham WT, Singh JP, et al. Cardiac-resynchronization therapy in heart failure with a narrow QRS complex. N Engl J Med 2013;369:1395-405.

10. Epstein AE, DiMarco JP, Ellenbogen KA, et al. ACC/AHA/HRS 2008 guidelines for device-based therapy of cardiac rhythm abnormalities: A report of the American College of Cardiology/ American Heart Association Task Force on Practice Guidelines (Writing Committee to Revise the ACC/AHA/NASPE 2002 Guideline Update for Implantation of Cardiac Pacemakers and Antiarrhythmia Devices) developed in collaboration with the American Association for Thoracic Surgery and Society of Thoracic Surgeons. J Am Coll Cardiol 2008;51:e1-62.

11. Abdel-Qadir HM, Tu JV, Austin PC, Wang JT, Lee DS. Bundle branch block patterns and long-term outcomes in heart failure. Int J Cardiol 2011;146:213-8.

12. Garrigue S, Reuter S, Labeque JN, et al. Usefulness of biventricular pacing in patients with congestive heart failure and right bundle branch block. Am J Cardiol 2001;88(1436-41):A8.

13. Chandra R, Zolty R, Palma E. A left hemiblock improves cardiac resynchronization therapy outcomes in patients with a right bundle branch block. Clin Cardiol 2010;33:89-93.

14. Hawkins NM, Petrie MC, MacDonald MR, Hogg KJ, McMurray JJ. Selecting patients for cardiac resynchronization therapy: Electrical or mechanical dyssynchrony? Eur Heart J 2006;27: 1270-81.

15. O'Connell JW, Schreck C, Moles M, et al. A unique method by which to quantitate synchrony with equilibrium radionuclide angiography. J Nucl Cardiol 2005;12:441-50.

16. Zhou W, Jiang Z, Chen J, Garcia EV, Li D. Development and validation of a phase analysis tool to measure interventricular mechanical dyssynchrony from gated SPECT MPI. J Nucl Cardiol 2016. doi:10.1007/s12350-016-0503-6.

17. Malhotra S, Pasupula D, Khanna M, Soman P. Is left bundle branch block related to the mechanism of left ventricular dyssynchrony? J Am Coll Cardiol 2014;63:A1121.

18. Feigenbaum $\mathrm{H}$. Role of M-mode technique in today's echocardiography. J Am Soc Echocardiogr 2010;23(240-57):335-7. 